\title{
Молдаванов С.Ю. \\ Прогнозирование длительной прочности полых сфер при действии внешнего гидростатического давления
}

Кубанский государственный технологический университет (Россия, Краснодар)

doi:10.18411/spc-12-02-2018-18

idsp: 000001:spc-12-02-2018-18

Рассмотрим основные положения статистической модели накопления повреждений и разрушения твердых тел, используемой в данной работе [1].

1. Разрушение твердых тел при действии внешних сил представляет собой термодинамически необратимый вероятностный процесс накопления повреждений развивающийся во времени на различных уровнях структурной организации материала.

2. Образование, накопление и развитие повреждений различных типов на всех уровнях разрушения происходит в результате накопления необратимых разрывов связей. В процессе разрыва связи атомы преодолевают некоторый энергетический барьер. Величина энергетического барьера зависит от средней удельной внутренней энергии, которая полностью определяет уравнения состояния и физико-механические свойства макрочастицы твердого тела.

3. Ввиду того, что микроструктура реальных материалов содержит дефекты и неоднородности, удельная внутренняя энергия неоднородно распределяется по связям в пределах макрочастицы. Начало и развитие процесса разрушения во времени в высокой степени локализовано и определяется функциями распределения минимальных времен разрушения (разрыва связей, разрушения макрочастицы, разрушение тела) на каждом уровне, которые могут быть вычислены на основании концепции наислабейшего звена с переменным числом звеньев.

Пусть случайная величина $t$ представляет время необратимого разрыва связей (атомных, молекулярных) в данной точке тела $\boldsymbol{X}$. Пусть $P(t, \boldsymbol{X})$ представляет функцию распределения времен необратимого разрыва связей, а $p(t, \boldsymbol{X})_{-}$ представляет плотность распределения времен необратимого разрыва связей, которые удовлетворяют естественным условиям:

$$
\int_{t_{0}}^{t^{*}} p(t, \boldsymbol{X}) d t=1, \quad P(t, \boldsymbol{X})=\int_{t_{0}}^{t} p(t, \boldsymbol{X}) d t,
$$

где $\boldsymbol{X}$ - вектор лагранжевых координат точек тела, $t_{0}-$ наименьшее и $t^{*}-$ наибольшее время разрыва связи.

Вычислим плотность распределения времен необратимого разрыва связей $p(t, \boldsymbol{X})[1]$. В процессе разрыва связей атомы или молекулы, совершая колебания около своего равновесного положения, преодолевают некоторый энергетический барьер. Если величина этого барьера равна $U_{m}-\gamma \hat{U}$, то колеблющийся атом в состоянии преодолеть его лишь за какую-то часть периода колебаний, пропорциональную больцмановскому распределению вероятностей тепловых флуктуаций в зависимости от энергетического состояния атома [2]. Пусть частота колебаний атома равна $1 / \tau_{0}$. Тогда при обычных тепловых колебаниях относительно положения равновесия атом $1 / \tau_{0}$ раз в секунду «ударяется» о потенциальный барьер. Большую часть времени энергия атома мала для преодоления энергетического барьера, но иногда (в соответствии с распределением Больцмана) в результате флуктуаций энергия атома может превысить высоту энергетического барьера и происходит разрыв 
связи. Тогда с учетом действия внешних сил вероятность разрыва связи в единицу времени будет равна [1]:

$$
\omega=\tau_{0}^{-1} \exp \left[-\left(U_{*}-\gamma U\right) / k T\right],
$$

где $\hat{U}$ - удельная внутренняя энергия в точке тела $\boldsymbol{X}$.

Выражение для удельной внутренней энергии $\hat{U}$ можно записать в зависимости от тензора напряжений $\tilde{S}(\tau, \boldsymbol{X})$ :

$$
U=U[\tilde{S}(\tau, \boldsymbol{X}), T(\tau, \boldsymbol{X})], t_{0} \leq \tau \leq t .
$$

где $\tau_{0}$ - период тепловых колебаний атомов (молекул), $U_{*}=\left(U_{m}-\gamma\left\langle U_{0}\right\rangle\right)$ энергетический барьер в отсутствие действия внешних сил, $\left\langle U_{0}\right\rangle-$ средняя удельная внутренняя энергия, приходящаяся на одну связь в отсутствие действия внешних сил, $U_{m}$ - энергия сублимации связи, $k$ - постоянная Больцмана, $T(\tau, \boldsymbol{X})$ - абсолютная температура, $\gamma=k_{0} \gamma_{c}, \gamma_{c}$ - часть объема тела, приходящаяся на одну связь, $k_{0} \geq 1-$ коэффициент неоднородности распределения внутренней энергии между связями в макрочастице тела.

В макрочастице твердого тела разрывы и восстановления связей происходят одновременно. Для безопасного уровня внутренней энергии $\hat{U}=\left\langle U_{0}\right\rangle+U_{0}$, вероятность (2) разрыва связей и их восстановления в единицу времени одинаковы и равны:

$$
\begin{aligned}
& \omega_{0}=\tau_{0}^{-1} \exp \left[-\left(U_{*}-\gamma U_{0}\right) / k T\right] \\
& U_{0}=U_{0}[\tilde{S}(\tau, \boldsymbol{X}), T(\tau, \boldsymbol{X})], 0 \leq \tau \leq t_{0} .
\end{aligned}
$$

В этом случае общее число связей в макрочастице твердого тела остается неизменным и процесс накопления повреждений и разрушения не наблюдается. При условии, когда $U>U_{0}$, вероятность актов разрыва связей в единицу времени $\omega$, определяемая по выражению (2), будет больше, чем вероятность их восстановления $\omega_{0}$ (4). Тогда в результате необратимого разрыва связей происходит образование и накопление субмикроповреждений макрочастицы тела. Следовательно, вероятность необратимого процесса разрыва связей в единицу времени равна:

$$
p(t, \boldsymbol{X})=\omega-\omega_{0}=B\left\{\exp \left[\gamma\left(U-U_{0}\right) / k T\right]-1\right\},
$$

где $B=\tau_{0}^{-1} \exp \left[-\left(U_{*}-\gamma U_{0}\right) / k T\right]$.

Полученное выражение (6) содержит многочисленные частные случаи. В случае деформации термоупругих тел при условии $T=T_{0}=$ const внутренняя энергия макрочастицы твердого тела является функцией тензора напряжений, и, следовательно, $p(t, \boldsymbol{X})=p(\boldsymbol{X})$. Используя выражения (1), (4), (5) и (6), получаем:

$$
\begin{gathered}
P(\boldsymbol{X})=\left(t-t_{0}\right) /\left[t^{*}(\boldsymbol{X})-t_{0}\right], \\
p(\boldsymbol{X})=\left[t^{*}(\boldsymbol{X})-t_{0}\right]^{-1}, \\
t^{*}(\boldsymbol{X})-t_{0}=B^{-1}\left\{\exp \left[\gamma\left(U-U_{0}\right) / k T_{0}\right]-1\right\}^{-1}, \\
B=\tau_{0}^{-1} \exp \left[-\left(U_{*}-\gamma U_{0}\right) / k T_{0}\right] .
\end{gathered}
$$

где $U_{0}=U_{0}\left[\tilde{S}_{0}(\boldsymbol{X}), T_{0}\right], \widetilde{S}_{0}(\boldsymbol{X})$ - тензор безопасных напряжений. 
Удельная внутренняя энергия твердого [3] тела определяется по формуле

$$
U=\frac{1+v}{2 E} \sigma_{i j} \sigma_{i j}-\frac{v}{2 E} \sigma_{k k}^{2}+T \alpha \sigma_{k k},
$$

где $T$ - температура; $E$ - модуль упругости материала; $\alpha$ - коэффициент линейного теплового расширения материала; $\sigma_{i j}-$ компоненты тензора напряжений; $v-$ коэффициент Пуассона.

Воспользуемся допущением о естественном состоянии твердого тела, в соответствие с которым при отсутствии внешних воздействий напряжения и деформации в твердом теле не возникают и, следовательно,

$$
\exp \frac{\gamma U_{0}}{k T(t)}=1 .
$$

Тогда наибольшее время разрушения макрочастицы твердого тела $t^{*}$ будет стремиться к бесконечности при выполнении следующего условия

$$
\frac{1+v}{2 E} \sigma_{i j} \sigma_{i j}-\frac{v}{2 E} \sigma_{k k}^{2}+T \alpha \sigma_{k k}=0 .
$$

Условие (12) будет выполняться, если первый инвариант напряженного состояния $\sigma_{k k}<0$, что соответствует сжатию материала. Из полученного условия (12) в работах [4] и [5] было получено выражение для вычисления безопасных напряжений в термоупругих телах для случая двухосного сжатия $\left(\sigma_{11}=\sigma_{22}=\right.$ const $\left.=-\sigma\right)$ :

$$
\sigma_{0}^{(2)}=\frac{2 E \alpha T}{1-v} .
$$

Рассмотрим сферическую оболочку (рис. 1), нагруженную внешним гидростатическим давлением. Воспользуемся безмоментной теорией оболочек В.З. Власова [6]. Действие внешнего гидростатического давления $p$ на замкнутую сферическую приводит к возникновению в ее нормальных и меридиональных сечениях сжимающих сил $N_{1}$ и $N_{2}$. Эти усилия относятся к единице длины дуги контура соответствующего сечения и постоянны для любой точки сферы:

$$
N_{1}=N_{2}=\frac{p R}{2} .
$$

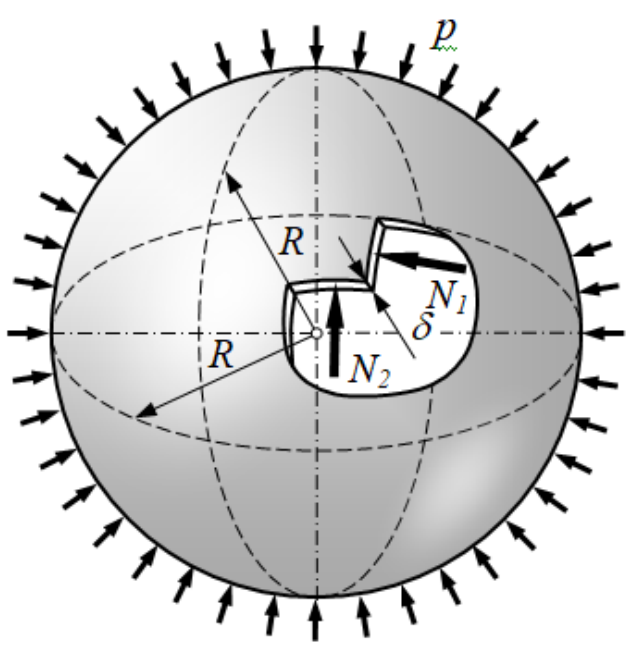

Рисунок 1. Сферическая оболочка 
Следовательно, материал сферической оболочки работает в условиях двухосного сжатия. Приравнивая выражения (14) и (16), получаем формулу для вычисления безопасной величины внешнего гидростатического давления:

$$
p_{0}=\frac{4 E \alpha T \delta}{(1-v) R}
$$

где $\delta$ - толщина оболочки, $R$ - радиус кривизны серединной поверхности.

В работе [7] были приведены данные экспериментальных исследований прочности замкнутых сферических оболочек, изготовленных из стекла марки МКР-1. Оболочки собирались из двух полусферических блоков и стыковались с помощью эпоксидного клея-компаунда. Стекло марки МКР-1 имеет следующие физикомеханические характеристики: коэффициент Пуассона $v=0,22$; модуль упругости $E=76$ ГПа; коэффициент линейного теплового расширения $\alpha=4 \cdot 10^{-6}$ град ${ }^{-1}$. Радиус срединной поверхности оболочки $R=94,5$ мм, толщина стенки $h=11$ мм. Температура $T=293^{\circ}$ К. Используя выражение (17), вычислим величину безопасного гидростатического давления для сферических оболочек из стекла марки МКР-1

$$
p_{0}=\frac{4 E \alpha T \delta}{(1-v) R}=\frac{4 \cdot 76 \cdot 10^{3} \cdot 4 \cdot 10^{-6} \cdot 293 \cdot 11 \cdot 10^{-3}}{(1-0,22) \cdot 94,5 \cdot 10^{-3} \cdot}=53,17 \text { МПа. }
$$

Составные стеклянные оболочки [7] подвергались следующим видам испытаний: кратковременному однократному гидростатическому давлению вплоть до разрушения; повторно-статическому нагружению и длительному нагружению при действии статической нагрузки.

При кратковременном нагружении разрушились 17 оболочек, которые отличались качеством изготовления, тщательностью подбора стеклоэлементов и точностью сборки. Результаты кратковременных испытаний свидетельствуют о значительном влиянии конструктивных и технологических факторов на прочность составных оболочек. Оболочки, разрушившиеся при внешнем давлении 59-90 МПа, имели максимальные несовершенства геометрии в виде радиальных смещений кромок в стыке и увеличенную толщину клеевого шва. Торцы стеклоэлементов остальных оболочек предварительно обрабатывались алмазным инструментом, что позволило значительно уменьшить толщину клеевого шва и существенно повысить их несущую способность (рис. 2).

Оболочки, предназначенные для повторно-статических испытаний [7], были разделены на две группы. Первая группа подвергалась повторно-статическим испытаниям при уровне максимального давления в цикле 59 МПа с длительностью цикла 17 минут. После 100 циклов нагружения испытываемые оболочки никаких видимых повреждений не получили. Затем эти оболочки подвергали повторному циклическому нагружению при давлении в цикле 98 МПа. Дополнительные испытания показали ограниченную работоспособность стеклянных оболочек в таких условиях нагружения (разрушение наблюдалось в 1-10 циклах). 


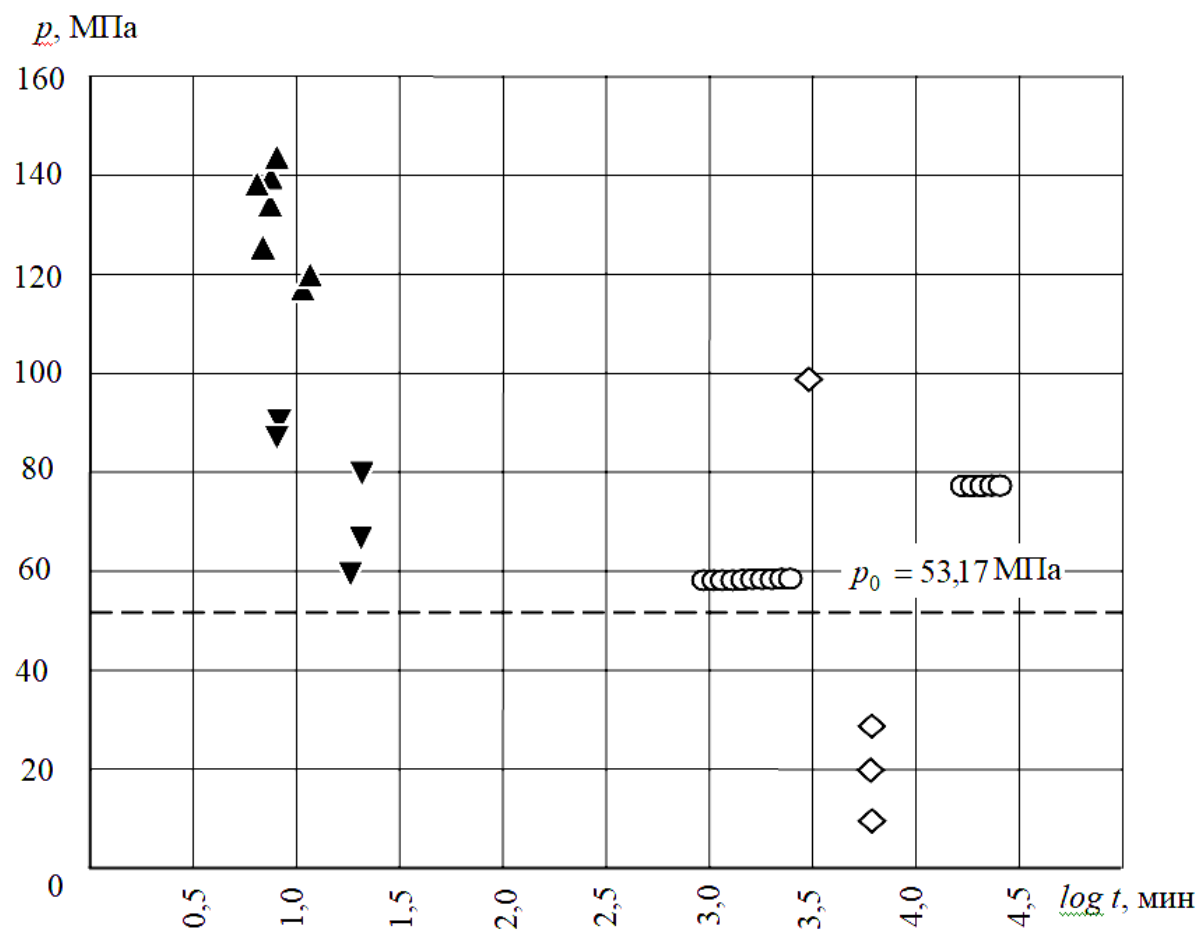

Рисунок 2. Экспериментальные данные по исследованию прочности стеклянных сфер, нагруженных гидростатическим давлением

- оболочки, разрушившиеся при однократном нагружении, с дефектами геометрии и увеличенной толщиной клеевого шва;

- оболочки, разрушившиеся при однократном нагружении, с улучшенной геометрией и уменьшенной толщиной клеевого шва;

- оболочки, не разрушившиеся в результате повторно-статических испытаниях;

- оболочки, не разрушившиеся при длительном действии статической нагрузки

Вторая группа оболочек подвергалась последовательному 1850-кратному повторно-статическому нагружению при максимальном давлении 78 МПа с последующим 100-кратным нагружением до 59 МПа. Эти оболочки получили незначительные повреждения в виде мелких сколов стекла и остались пригодными для дальнейших испытаний (рис. 2).

На следующем этапе эксперимента [7] определялось сопротивлению разрушению составных оболочек при длительном действии статической нагрузки. Выдержка стеклянных оболочек под постоянным внешним давлением составляла 50 или 100 часов. Непрерывное однократное нагружение оболочки в течении 50 часов внешним гидростатическим давлением 98 МПа не привело к образованию каких-либо видимых локальных повреждений стеклоэлементов или изделия в целом (рис. 2). Нагружение составных стеклянных оболочек в течении 300 часов ступенчатым повышением внешнего давления 10, 20 и 29 МПа (по 100 часов каждым) также не вызвало никаких повреждений.

Результаты экспериментальных исследований [7] хорошо согласуются с полученной формулой для определения безопасного уровня внешнего гидростатического давления при нагружении сфер.

$$
* * *
$$

1. Dunaev I.M. Statistical Theory of Fracture of Structural Materials at Damage Accumulation. Proc. of Joint FEFG/ICF Int. Conf. on Fracture of Eng. Mat. \& Structures. $\square$ Elsevier Applied Science, 1991, pp. $832 \square 837$.

2. Ландау Л.Д., Лившиц Е.М.Статистическая физика.- М.: Наука, 1964, 567 с.

3. Дунаев И.М. Разрушение эластомеров. В кн. Механика эластомеров. Межвузовский сб. Краснодар, Краснодар. политехн. ин-т, 1981, С. $24 \square 33$. 
4. Молдаванов С.Ю. Прогнозирование длительной прочности термоупругих тел при сжатии. Научные труды SWorld. 2013. Т. 3. № 2. С. 21-26.

5. Молдаванов С.Ю., Дунаев В.И. Вычисление предела длительной прочности неорганических стекол при сжатии. Политехнический вестник. 2013. № $1 \square$ 2. С. $13 \square 18$.

6. Власов В.З. Избранные труды. Общая теория оболочек. Т. 1. $\square$ М. : Издво АН СССР, 1962 . $\square 528$ стр.

7. Квитка А.Л., Дьячков И.И. Напряженное состояние и прочность оболочек из хрупких неметаллических материалов. $\square$ Киев: Наукова думка, 1983, 284 с.

\section{Мунин А.А., Бржозовский Б.М. \\ Исследование инварных покрытий получаемых наплавкой и их характеристики и свойства}

СГТУ им.Гагарина Ю.А. (Россия, Саратов)

doi:10.18411/spc-12-02-2018-19

idsp: 000001:spc-12-02-2018-19

\section{Аннотация}

Статья посвящена инварных покрытий их характеристикам и свойствам, нанесение их а поверхность с помощью наплавки. Предлагаемая технология нанесения наплавочных покрытий исключает применение дополнительных операций. По результатам испытаний сделаны выводы об эффективности нанесения данного сплава.

Ключевые слова: Инварные покрытия, инвары, наплавка покрытий, свойства, испытания, эффективность.

Актуальность темы. В условиях истощения сырьевых ресурсов, экологических и социально-экономических затруднений особую значимость обретают ресурсосберегающие технологии, которые все чаще сопровождаются освоением методов наплавки.

В настоящее время проблема срока службы деталей машин и агрегатов, их долговечности и надежности приобрела весьма большое значение. Срок службы быстроизнашивающихся деталей определяет рентабельность многих дорогостоящих машин. Поставленная задача решается тем, что в способе восстановления наплавкой поверхностей деталей, включающем наплавку плавящимся электродом на поверхность восстанавливаемой детали, перемещаемой относительно электрода, с образованием наплавочной ванны, подачу в наплавочную ванну присадочной проволоки, согласно предлагаемому решению присадочную проволоку, выполненную из интерметаллидов в частности никеля и нихрома, подают под острым углом к направлению подачи плавящегося электрода перед или после него по направлению относительного перемещения детали и на расстоянии от него, обеспечивающем сохранение интерметалидных элементов в наплавленном металле, при этом присадочную проволоку изолируют от тока.

В наплавочную ванну вводят одну или две дополнительные присадочные проволоки, которые располагают с противоположной стороны от первой присадочной проволоки относительно плавящегося электрода.

В наплавочную ванну вводят три дополнительные присадочные проволоки, одну из которых располагают со стороны первой, а две другие - с противоположной стороны относительно плавящегося электрода. При этом две присадочные проволоки, расположенные с одной стороны относительно плавящегося электрода, параллельны друг другу и расположены симметрично плоскости, параллельной вектору мгновенной скорости детали и перпендикулярной поверхности детали в точке контакта плавящегося электрода с поверхностью детали.

Наплавку осуществляют под слоем флюса. 